\title{
INSULIN + NUTRITION CONTROL FOR TIGHT CRITICAL CARE GLYCAEMIC REGULATION
}

\author{
J. Geoffrey Chase*, Jason Wong*, Jessica Lin*, Aaron LeCompte*, \\ Thomas Lotz*, Timothy Lonergan*, Michael Willacy*, \\ Christopher E. Hann* and Geoffrey M. Shaw**
}

*Centre for Bioengineering, University of Canterbury, Christchurch, New Zealand
**Department of Intensive Care Medicine, Christchurch Hospital, Christchurch, New Zealand

\begin{abstract}
A new insulin and nutrition control method for tight glycaemic control in critical care is presented from concept to clinical trials to clinical practice change. The primary results show that the method can provide very tight glycaemic control in critical care for a very critically ill cohort. More specifically, the final clinical practice change protocol provided 2100 hours of control with average blood glucose of $5.8+/-0.9$ $\mathrm{mmol} / \mathrm{L}$ for an initial 10 patient pilot study. It also used less insulin, while providing the same or greater nutritional input, as compared to retrospective hospital control for a relatively very critically ill cohort with high insulin resistance. Copyright $($ C) 2006 IFAC
\end{abstract}

Keywords: Biomedical Control, Control Algorithms, Non-Linear Models, Physiological Models, Physiology, Medical Systems.

\section{INTRODUCTION}

Hyperglycaemia is prevalent in critical care, and worsens outcomes, increasing the risk of severe infection, myocardial infarction, neuropathy, and multiple organ failure (Krinsley, 2003; Van den Berghe et al., 2001). Tight glucose control can reduce mortality by up to $45 \%$. However, insulinmediated control, is severely challenged in critical care by very high effective insulin resistance (McCowen et al., 2001; Mizock, 2001). Glycaemic reductions are thus limited by insulin effect saturation at high concentrations (Prigeon $e t$ al., 1996). Next, high glucose content nutritional support exacerbates hyperglycaemia (Weissman, 1999) and studies with lower glucose nutrition alone saw large reductions in glucose levels (Patino et al., 1999).

\section{MODELS and METHODS}

This research presents the development of an insulin nutrition method for tight glycaemic control in critical care. First, a virtual cohort is used to develop the method in simulation. Second, the methods are tested in limited proof of concept clinical trials. Finally, a paper-based method that mimics the computerised controller is introduced as a clinical practice change for long-term testing to validate the overall concept.

\subsection{Control Model}

Chase et al (2005a) used a system model that that included insulin utilisation, losses and saturation.

$\dot{G}=-p_{G} G-S_{I}\left(G+G_{E}\right) \frac{Q}{1+\alpha_{G} Q}+P(t)$

$\dot{Q}=-k Q+k I$

$\dot{I}=-\frac{n I}{1+\alpha_{I} I}+\frac{u_{e x}(t)}{V}$

$P\left(t_{i}<t<t_{i+1}\right)=\bar{P}_{i+1}+\left(\bar{P}_{i}-\bar{P}_{i+1}\right) e^{-k_{p d}\left(t t_{i}\right)}$ where $\bar{P}_{i+1}<\bar{P}_{i}$

$P\left(t_{i}<t<t_{i+1}\right)=\bar{P}_{i+1}+\left(\bar{P}_{i}-\bar{P}_{i+1}\right) e^{-k_{p r}\left(t-t_{i}\right)}$ where $\bar{P}_{i+1}>\bar{P}_{i}$

where $G(t)[\mathrm{mmol} / \mathrm{L}]$ is the plasma glucose above an equilibrium level, $G_{E}[\mathrm{mmol} / \mathrm{L}] . I(t)[\mathrm{mmol} / \mathrm{L}]$ is plasma insulin concentration resulting from exogenous insulin input, $u_{e x}(t)[\mathrm{mU} / \mathrm{min}] . Q(t)$ $[\mathrm{mU} / \mathrm{L}]$ is interstitial insulin concentration and $k$ [1/min] accounts for the effective life of insulin in the system. Patient endogenous glucose clearance and insulin sensitivity are $p_{G}[1 / \mathrm{min}]$ and $S_{I}$ 
[L/(mU.min)], respectively. $V[\mathrm{~L}]$ is the insulin distribution volume and $n[1 / \mathrm{min}]$ is the constant first order decay rate for insulin from plasma. Total plasma glucose input is denoted $P(t)$ [mmol/(L.min)]. $k_{p r}$ is the rise rate of rate of plasma glucose input from enterally administered feed $[1 / \mathrm{min}] . k_{p d}$ is the decay rate of rate of glucose input into plasma from enterally administered feed [1/min]. $\bar{P}_{i}, \bar{P}_{i+1}$ are stepwise consecutive enteral glucose feed rates [mmol/L.min]. Michaelis-Menten functions model saturation, with $\alpha_{I}[\mathrm{~L} / \mathrm{mU}]$ for the saturation of plasma insulin disappearance, and $\alpha_{G}[\mathrm{~L} / \mathrm{mU}]$ for the saturation of insulin-dependent glucose clearance. In this research, $k, n, \alpha_{G}, \alpha_{I}$ and $V$ are identified from generic population values. Details of the model, its development, and control analyses presented for it and similar models models can be found in (Chase, et al., 2005a).

\subsection{Control Method}

In this study, non-steady stepwise enteral glucose fluxes are employed for control and modelled using the 2-compartment model in Eqs. (4-5). The exponential rates for total glucose rate of appearance (GRa) rise $\left(k_{p r}\right)$ and decay $\left(k_{p d}\right)$ model the effect of transient net hepatic glucose output and glucose disposal. Impaired splanchnic and peripheral glucose uptake imply a slow decay rate in total GRa following nutritional feed reduction (Kiwanuka et al., 2001). Conversely, the rate of peripheral appearance of oral glucose is approximately equal to the intestinal absorption rate, implying a rapid rise following a nutritional increase (Radziuk et al., 1978). Thus, $k_{p r}$ and $k_{p d}$ are set to $0.0347 \mathrm{~min}^{-1}$ and $0.0068 \mathrm{~min}^{-1}$ (half-lives of 20 and $100 \mathrm{mins}$ ) to reflect this data.

The controller targets $10-15 \%$ hourly glycaemic reduction to $5 \mathrm{mmol} / \mathrm{L}$ using a combination of insulin bolus, infusion and/or feed rate change. The goal is blood glucose in the $4-6 \mathrm{mmol} / \mathrm{L}$ band. Thus, insulin sensitivity, $S_{I}$, is fitted from the prior hours' data before each intervention (Hann et al., 2005) and endogenous clearance, $p_{G}$, is set to $0.01 \mathrm{~min}^{-1}$, a value found to be insensitive across this type of cohort (Hann et al., 2005). Finally, the required combination of insulin bolus, insulin infusion rate and/or nutritional feed rate to achieve the hourly target glucose is determined iteratively using the updated $S_{I}$, value and Eqs. (1)-(5).

\subsection{Virtual Cohort and Simulated Trials}

The patient cohort includes 17 patients from a 201 patient data audit plus 2 patients from a hyperglycaemia control clinical trial (Chase et al., 2005b). It represents a general cross-section of ICU population, in medical subgroup, APACHE II score, age, sex and mortality. Each record has glucose measurements every $3 \mathrm{~h}$ or less. The average length is 3.9 days (range: 1.4-18.8). The cohort details are in Table 1. Ethical consent was granted by the Canterbury Ethics Committee.

Virtual trials use the retrospective fitted patient profiles of $S_{I}$ and $p_{G}$, to simulate physiological patient response. It assumes these parameters are independent of the control inputs administered, creating a virtual patient response for any glucose or insulin inputs. Normally distributed error of $\pm 7 \%$ is added to measured glucose values to include typical glucometer measurement error.

Table 1: Long-Term Virtual Trial Patient Cohort

\begin{tabular}{|c|c|c|c|c|c|c|}
\hline $\begin{array}{l}\text { Patient } \\
\text { number }\end{array}$ & $\begin{array}{l}\text { Medical } \\
\text { subgroup }\end{array}$ & $\begin{array}{l}\text { Apache II } \\
\text { score }\end{array}$ & Age & Sex & Mortality & Diabetes \\
\hline 1 & Sepsis & 17 & 56 & $\mathrm{M}$ & & Type 2 \\
\hline 2 & Sepsis & 24 & 64 & M & & \\
\hline 24 & Other medical & 25 & 47 & M & $\mathrm{Y}$ & Type 1 \\
\hline 87 & Other medical & 26 & 62 & $\mathrm{~F}$ & & \\
\hline 130 & Trauma & 11 & 21 & M & & Type 1 \\
\hline 229 & Cardiac & 15 & 73 & $\mathrm{~F}$ & & \\
\hline 289 & Cardiac & 18 & 70 & M & & \\
\hline 468 & General surgical & 32 & 76 & M & & \\
\hline 484 & Other medical & 34 & 30 & $\mathrm{~F}$ & & \\
\hline 486 & General surgical & 22 & 76 & $\mathrm{~F}$ & & Type 2 \\
\hline 519 & General surgical & 29 & 69 & M & & Type 2 \\
\hline 554 & Other medical & 26 & 20 & $\mathrm{~F}$ & & Type 1 \\
\hline 666 & Cardiac & 8 & 44 & $\mathrm{~F}$ & & Type 2 \\
\hline 847 & Other medical & 17 & 67 & $\mathrm{~F}$ & & \\
\hline 1016 & General surgical & 20 & 37 & $\mathrm{~F}$ & & Type 2 \\
\hline 1025 & Pulmonary & 36 & 48 & M & & Type 2 \\
\hline 1090 & General surgical & Unknown & 37 & $\mathrm{~F}$ & & \\
\hline 1099 & Pulmonary & Unknown & 24 & M & $\mathrm{Y}$ & \\
\hline 1125 & Other medical & Unknown & 72 & $\mathrm{~F}$ & Y & \\
\hline
\end{tabular}

Each patient is tested using the control method. Results are compared to retrospective hospital data and an insulin-only control protocol (Chase et al., 2005a). The control method uses three basic steps:

- Measure glucose every 30 minutes

- Every hour fit SI based on prior hours data

- Determine the insulin and nutritional changes to meet target reduction

Frequent measurement ensures tight control and safety. Hence, these trials are used for proof of concept testing of the insulin plus nutrition control concept. Less frequent measurement would be required for long-term clinical care.

\subsection{Clinical Trials Method}

The methods developed virtually are tested in short 10-hour proof-of-concept trials and one 24-hour trial. Inclusion criteria: in situ enteral feeding tube; random glucose level $>8 \mathrm{mmol} / \mathrm{L}$; age $>16$ years; and an in situ cannula. Exclusion criteria: delayed gastric emptying; moribund; neuromuscular blockade; and morbid obesity $\left(\mathrm{BMI}>35 \mathrm{kgm}^{-2}\right)$.

Patients are fed enterally with RESOURCETM Diabetic (Novartis Medical Nutrition, USA) up to $700 \mathrm{kcal} /$ day of glucose using a Ross Products Patrol Enteral Pump (Abbott Laboratories, Abbott Park, Illinois, U.S.A.). Actrapid ${ }^{\mathrm{TM}}$ insulin (Novo Nordisk, Bagsvaerd, Denmark) is infused with a 3500 syringe pump (Graseby Medical Limited, Colonial Way, Herts, UK). Ethical approval was obtained from the Canterbury Ethics Committee.

The overall trial protocol is shown in Figure 1. During the 2-hour pre-trial period, the insulin 
infusion is kept constant to estimate the onboard insulin level in steady state. The blood glucose level at $0900 \mathrm{~h}$ is taken as the equilibrium blood glucose, $G_{E}$. At $0900 \mathrm{~h}$, feed rate is decreased by $30-40 \%$ depending on current glucose level and feed rate as an initial challenge. Hourly glucose targets are set for a $10-15 \%$ reduction to a $5 \mathrm{mmol} / \mathrm{L}$ minimum. Insulin sensitivity, $S_{I}$, is reevaluated every hour using the prior hours' data. The controller prescribes insulin bolus size, insulin infusion rate, and feed rate to achieve the target.

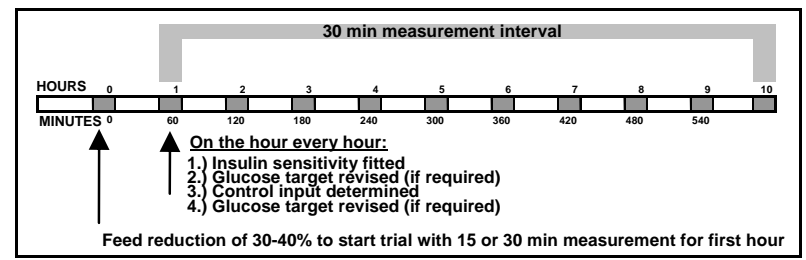

Fig.1: Clinical Trial Procedure

Note that glucose is only measured hourly for the 24-hour trial. This trial tests the ability of the controller to use less frequent measurements as a final step towards clinical, long-term testing.

Insulin is limited to $6 \mathrm{U} / \mathrm{hr}$ to minimise saturation and saturated, ineffective insulin is limited to $30 \mathrm{mU} / \mathrm{L}$. The minimum feed rate is $280 \mathrm{kcal} /$ day of glucose or $40 \%$ of maximum for a total caloric intake of $778 \mathrm{kcal} /$ day (Novartis, 2005). This level exceeds the level found to avoid increased risk of bloodstream infections (Rubinson et al., 2004).

\subsection{Clinical Trials Cohort}

The clinical trial patient cohort $(n=8)$ represents a heterogeneous cross-section in age and sex, as shown in Table 2. The median APACHE II score is 23 with inter-quartile range $[19,25]$. The mean age is $64.8 \pm 7.8$ years.

Table 2: Clinical Trial Patient Cohort

\begin{tabular}{|c|c|c|c|c|c|c|c|c|c|c|}
\hline $\begin{array}{l}\begin{array}{l}\text { Patient } \\
\text { number }\end{array} \\
\text { number }\end{array}$ & $\begin{array}{l}\text { Medical } \\
\text { subgroup }\end{array}$ & $\begin{array}{l}\text { APACHE II } \\
\text { score }\end{array}$ & $\begin{array}{l}\text { APACHE II } \\
\text { ROD (\%) }\end{array}$ & APACHE III & SAPS II & $\begin{array}{c}\text { SAPS II } \\
\text { ROD }(\%)\end{array}$ & Age & Sex & Mortality & Diabetes \\
\hline 1 & Sepsis & 17 & 14.3 & 40 & 15 & 2 & 56 & M & $\mathrm{N}$ & Type 2 \\
\hline 2 & Sepsis & 24 & 49.7 & 59 & 35 & 16.7 & 64 & M & $\mathrm{N}$ & \\
\hline 3 & Pulmonary & 31 & 73.3 & 85 & 45 & 34.8 & 60 & M & $\mathrm{N}$ & \\
\hline 4 & Sepsis & 26 & 59.7 & 91 & 62 & 71.9 & 75 & $\mathrm{~F}$ & $\mathrm{~N}$ & \\
\hline 5 & Sepsis & 21 & 33.2 & 58 & 34 & 15.3 & 73 & M & $\mathrm{N}$ & Type 2 \\
\hline 6 & Other medical & 17 & 14.3 & 44 & 44 & 32.6 & 57 & M & $\mathrm{N}$ & \\
\hline 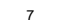 & General surgica & 23 & 62.3 & 84 & 57 & 61.9 & 73 & $\mathrm{~F}$ & $\mathrm{~N}$ & Type 2 \\
\hline
\end{tabular}

\subsection{Long-term Testing - The SPRINT Protocol}

The clinical methods are developed and tested, first virtually and then in short proof of concept case studies. The final step is long-term clinical testing of this nutrition and insulin control approach. However, the measurement frequency must be reduced to 1-2 hourly for clinical ease of use. In addition, the methods must be removed from a computer and made paper based for easy uptake by clinical staff.

The SPRINT (Specialised Relative Insulin Nutrition Tables) protocol is designed to nearly exactly mimic the computerised trial protocol, as an easy-to-use equivalent. It consists of two wheels dedicated to enteral nutrition and insulin bolus administration, as shown in Figure 2. Instructions are printed on the wheels and hourly blood glucose measurements are used to determine the next hour's intervention.

The instructions on the "Feed Wheel" are used to determine the rate of feed as a percentage of the patient's clinically determined goal feed. The result is based on the previous hour's feed level, the current blood glucose concentration and whether blood glucose is rising or falling. The percentage goal feed is converted into an absolute feed rate (in $\mathrm{ml} / \mathrm{hr}$ ) using a patient-specific conversion sticker. The "Insulin Wheel" is then used to determine the insulin bolus size based on the previous insulin bolus size, the current blood glucose level and whether the blood glucose has decreased by more than $1.5 \mathrm{mmol} / \mathrm{L}$. The method is effectively fully automated once clinical staff take the hourly glucose measurement.

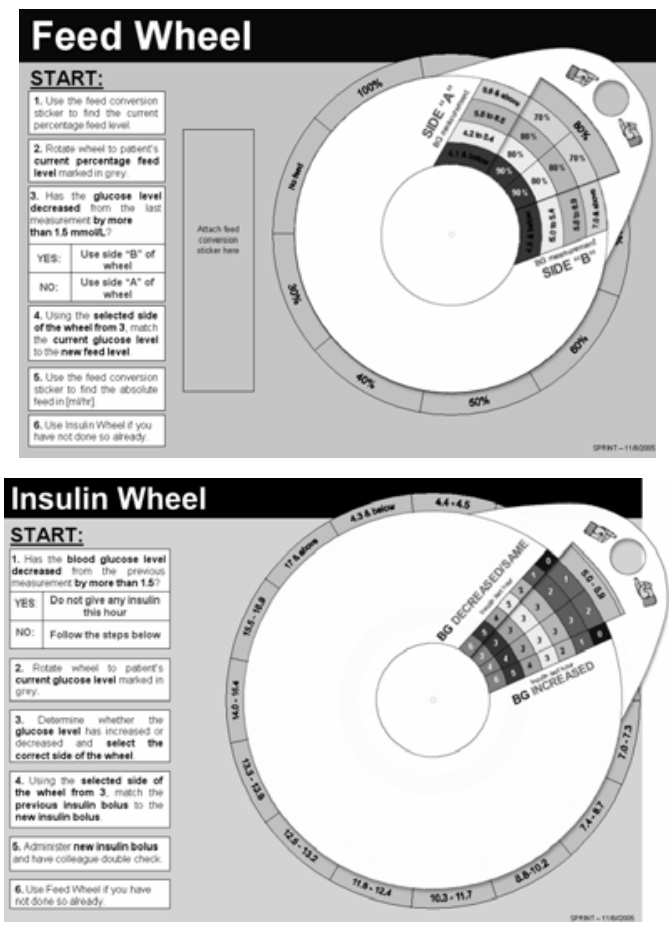

Fig.2: SPRINT feed and insulin wheels.

Hourly blood glucose measurements are used to ensure tight control. Two-hourly measurements are used when the patient is stable, defined as 3 consecutive measurements in the 4-6 mmol/L band. For two-hourly measurements, the feed rate is maintained and the same insulin bolus administered on the hour between measurements. Two-hourly measurements are continued until the patient leaves the $4-6 \mathrm{mmol} / \mathrm{L}$ band. SPRINT is stopped when the patient is stable, and normoglycaemic, defined as 6 or more hours in the 4-6.1 $\mathrm{mmol} / \mathrm{L}$ band, with over $80 \%$ of goal feed rate and a maximum of $2 \mathrm{U}$ per hour of insulin. Finally, insulin is always administered via bolus for patient safety, avoiding infusions being left on. 
The specific wheel layout resulted from extensive consultation. ICU staff were proficient in minutes and reported the system as very easy to use. A nursing survey reported that 25 of 27 respondents viewed the wheels as satisfactory or better, with 13 rating it very good or higher. The covered wheel reduces table complexity, which reduces error.

\section{RESULTS and DISCUSSION}

\subsection{Virtual Trial Results}

Figure 3 shows Patient 87 from retrospective hospital data, the insulin-only protocol (Chase et al., 2005a), and the variable feed and insulin protocol developed. Tight control in the 4$6 \mathrm{mmol} / \mathrm{L}$ desired band is clear with the variable nutrition protocol compared to the other protocols. The total insulin administered by the variable nutrition protocol is $38.5 \%$ less than the insulinonly protocol (410.5U versus $667.0 \mathrm{U})$. From the retrospective data, the total insulin infused was 248.0U, indicating another source of poor control. Time spent in the desired $4-6 \mathrm{~mol} / \mathrm{L}$ band was $89 \%$ versus $21.8 \%$ for the insulin-only protocol and $10.7 \%$ for hospital control. Finally, the results are achieved with total enteral glucose administered identical to the retrospective patient data $(1284 \mathrm{~g}$ versus $1286 \mathrm{~g}$ ).

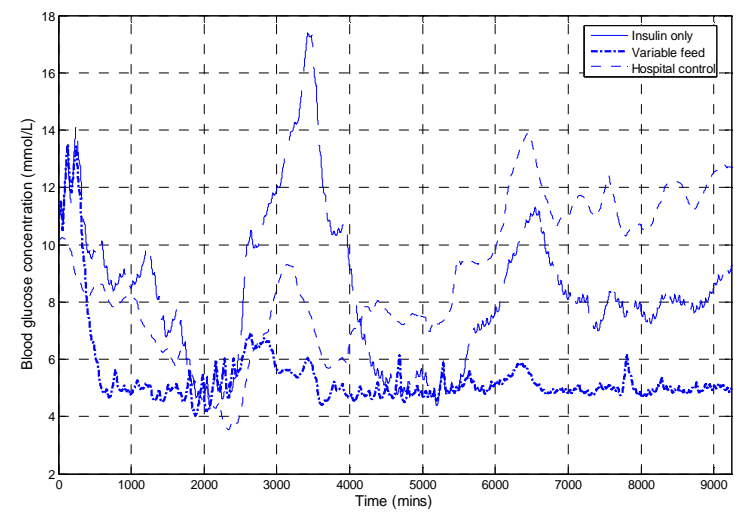

Fig.3: Patient 87 Virtual Trial Results

A summary of the results for all patients is shown in Table 3. The variable nutrition and insulin controller increased the time spent in the 4$6 \mathrm{mmol} / \mathrm{L}$ band by $240 \%$ compared to the insulinonly protocol and $312 \%$ versus the retrospective data. Time above $6 \mathrm{mmol} / \mathrm{L}$ is reduced by $231 \%$ and $237 \%$. No hypoglycaemic events occurred for any virtual trial protocols. Figure 4 summarises these results plotting percentage time in the 4$6 \mathrm{mmol} / \mathrm{L}$ band versus log mean fitted $S_{I}$.

Figure 4 shows that percentage time-in-band and mean blood glucose level decrease for all protocols with decreasing insulin sensitivity. With insulin alone, performance is highly dependent on the patients' effective insulin resistance $(R=0.90$, $p<0.001$ ) due to saturation limitations (Chase et al., 2005a). The variable feed rate and insulin protocol provided tighter blood glucose control across the range of observed insulin sensitivities with significantly higher time-in-band $(R=0.57$, $p<0.02)$. The insulin-only protocol only reached similar levels only at high insulin sensitivities, and with significantly more administered insulin. For hospital control, greater variation in blood glucose control was recorded, as expected $(R=0.49$, $p<0.04$ ), and showed tighter control than the insulin-only protocol only at low insulin sensitivities, where clinically selected feed reductions have affected the comparison.

Table 3: Blood Glucose Summary - Virtual Trials

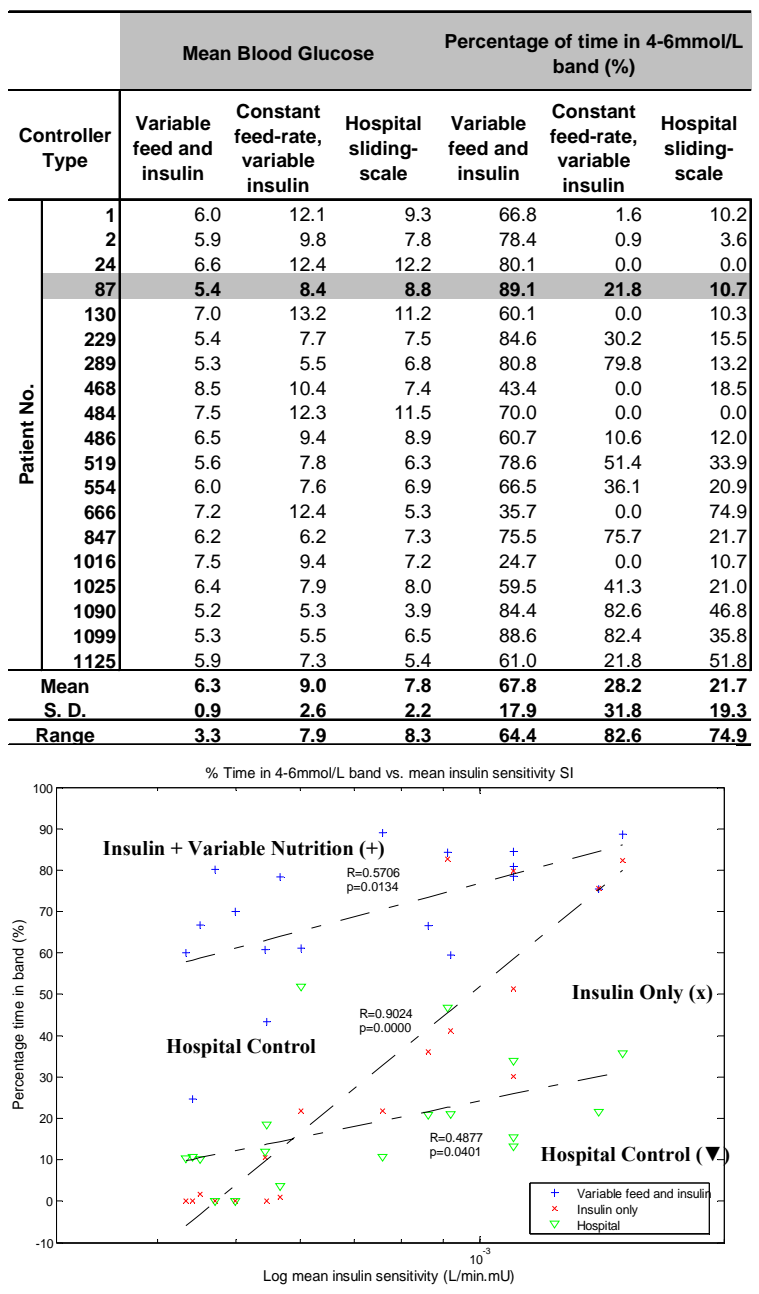

Fig.4: Mean Insulin Sensitivity, $S_{I}$, versus Time in the $4-6 \mathrm{mmol} / \mathrm{L}$ Band

In summary, these results all indicate the effectiveness of using nutrition as an added control input. In particular, high APACHE II score, very critically ill patients are generally highly insulin resistant. Thus, this added control path may represent the only means to maintain euglycaemia, as well as one that is not saturable as is the insulin path.

\subsection{Clinical Trial Results}

The main goal of the clinical trials was to prove the insulin and nutrition control concept by illustrating the potential for tight control. Tight control is shown by accurately reducing glucose 
levels to pre-set target values. Hence, the target error is the main performance criteria for evaluation.

The mean target error for all trials is $9.3 \%$ $(0.52 \mathrm{mmol} / \mathrm{L})$, absolute range $[0,2.9] \mathrm{mmol} / \mathrm{L}$, and $41.9 \%$ of targets are achieved within $\pm 5 \%$ with a mean target error of $2.6 \%(0.15 \mathrm{mmol} / \mathrm{L})$. Mean target error for errors $>5 \%$ is $14.3 \%(0.79 \mathrm{mmol} / \mathrm{L})$. Out of 86 targets, only seven had errors $>20 \%$, so that $90.7 \%$ of all measurements are within $\pm 20 \%$ of targets. More specifically almost $90 \%$ of target errors are explainable by measurement errors (Weitgasser et al., 1999). Outliers are attributed to significant and rapid changes in patient condition observed, such as atrial fibrillation. Model prediction errors at a $60 \mathrm{~min}$ glucose measurement frequency (Trial 8) were not statistically discernible from the other trials.

Figure 5 presents a bootstrapped linear regression model applied to the achieved and target glucose values using 6000 bootstrap samples. Also shown are the non-parametric $95 \%$ confidence intervals (CI) for the prediction of achieved glucose values for a given target. A correlation coefficient between 0.7695 and 0.8983 can be stated with $95 \%$ confidence.

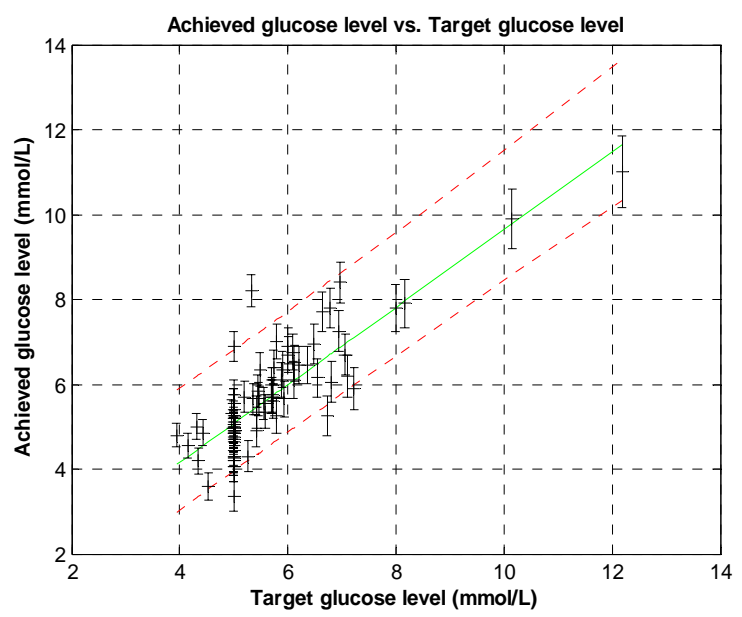

Fig.5: Target Error Summary

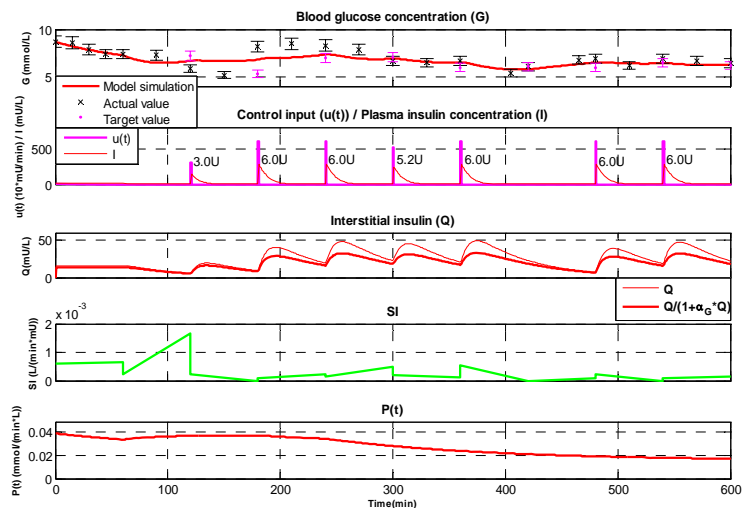

Fig.6: Patient 2 Clinical Trial Progression

Finally, Figure 6 shows a typical trial result for Patient 2. This trial highlights a common difficulty in critical care glycaemic control, the highly dynamic patient with rapidly evolving condition. The glucose measurement at $150 \mathrm{mins}$ was on course for the $5 \mathrm{mmol} / \mathrm{L}$ target at $180 \mathrm{mins}$. However, the recorded measurement at $180 \mathrm{mins}$ was $8.5 \mathrm{mmol} / \mathrm{L}$. The patient experienced atrial fibrillation at approximately 200mins, indicating that the change may have been due to adrenergic surge preceding the cardiac event. From that point, effective insulin resistance increased, requiring greater insulin input and feed rate reductions compared to the initial $60-120 \mathrm{mins}$. The controller adapted to this not uncommon event, tracking the glucose measurements accurately within the next hour and the $300 \mathrm{~min}$ target with $5.1 \%$ error.

\subsection{SPRINT Results}

The SPRINT protocol was implemented as a clinical practice change in the Christchurch Hospital ICU. The entry requirement was 2 successive random glucose measurements over 8 $\mathrm{mmol} / \mathrm{L}$. this limit ensures only the relatively more critically ill, and thus potentially more insulin resistant, patients are considered.

An initial pilot study of 10 patients was performed to test SPRINT over long term clinical care. The cohort had an average age of 54 (range: 44-80), average APACHE II score of 23 (range: 11-37) and an average APACHE III score of 70 (range: 34-108). There were 6 males and 4 females.

The total controlled time is 2103 hours with 1579 measurements indicating that $49.8 \%$ of the controlled time was on 2-hourly measurement as the patients were glycaemically stable. The average length of control for each patient was 210 hours (8.75 days), also indicating a significantly critically ill cohort.

The overall control results can be summarised:

- Average Blood Glucose $=5.8+/-0.9 \mathrm{mmol} / \mathrm{L}$

- Average Insulin = $2.5 \mathrm{U} /$ hour

- Average Feed Rate = 64\% (1279 kcal/day)

- Time Feed Rate $>50 \%$ of goal feed $=70 \%$

However, more relevant performance is time in glycaemic band and any hypoglycaemic events. The primary bands are the 4.-6.1 $\mathrm{mmol} / \mathrm{L}$ band defined by van den Berghe et al and the 4-7.75 $\mathrm{mmol} / \mathrm{L}$ band defined by Krinsley. More importantly, these bands are associated with reductions in mortality of $45 \%$ and $20-30 \%$ respectively, as well as significant reductions in other negative clinical outcomes. The overall glycaemic performance is thus summarised:

- Time in the 4-6.1 $\mathrm{mmol} / \mathrm{L}$ band $=64 \%$

- Time in the 4-7.0 $\mathrm{mmol} / \mathrm{L}$ band $=89 \%$

- Time in the 4-7.75 $\mathrm{mmol} / \mathrm{L}$ band $=97 \%$

- Number measurements $<4 \mathrm{mmol} / \mathrm{L}=23$ (1.5\%)

- Number Measurements $<3 \mathrm{mmol} / \mathrm{L}=0$

- Minimum Blood Glucose = $3.2 \mathrm{mmol} / \mathrm{L}$ 
Thus, the results indicate that SPRINT provided very tight glycaemic regulation. The values for time in the glycaemic bands from the landmark studies on hyperglycaemia and mortality are also very high. This latter result indicates that glycaemic levels were not only tightly controlled on average, but that their variation was also very limited. This result is backed up by the narrow 0.9 $\mathrm{mmol} / \mathrm{L}$ standard deviation. Overall, the results indicate that modulating nutrition and insulin, in combination with frequent measurement, can provide very tight control for a very critically ill cohort.

\section{CONCLUSIONS}

This paper has presented the development, from concept to clinical practice change, of a nutrition plus insulin control methodology for maintaining euglycaemia in critical care. The methods are developed from a model-based virtual study to proof of concept clinical trials using a model based controller. The control method is then made paper based through a system that effectively mimics the model-based control methods, and implemented as a clinical practice change. Thus, the overall methodology of using retrospective data through to clinical change is also presented as an approach to developing this type of model-based control therapy.

The overall results indicate that modulating nutrition and insulin is an effective approach to controlling hyperglycaemia in critical care. In particular, the more critically ill cohorts with higher APACHE II scores are typically more insulin resistant and this path may offer the only approach to lowering glucose levels to within a desired level or band. Also apparent in the need for higher measurement frequency to ensure that dynamic patients are well monitored and that inappropriate interventions of nutrition or insulin are not maintained when patient condition evolves significantly.

\section{REFERENCES}

Chase, J. G., G. M. Shaw, et al. (2005a). "Adaptive bolus-based targeted glucose regulation of hyperglycaemia in critical care." Med Eng Phys 27(1): 1-11.

Chase, J. G., X. W. Wong, et al. (2005b). Clinical Trials of Active Insulin and Nutrition Control in Critically Ill Patients. Proc. of the 12th International Conf on Biomedical Engineering (ICBME 2005), Singapore.

Hann, C. E., J. G. Chase, et al. (2005). "Integralbased parameter identification for longterm dynamic verification of a glucoseinsulin system model." Comput Methods Programs Biomed 77(3): 259-70.
Kiwanuka, E., R. Barazzoni, et al. (2001). "Glucose kinetics and splanchnic uptake following mixed meal ingestion in cirrhotic-diabetic subjects." Diabetes Nutrition \& Metabolism 14(6): 315-324.

Krinsley, J. S. (2003). "Association between hyperglycemia and increased hospital mortality in a heterogeneous population of critically ill patients." Mayo Clin Proc 78(12): 1471-1478.

McCowen, K. C., A. Malhotra, et al. (2001). "Stress-induced hyperglycemia." Crit Care Clin 17(1): 107-24.

Mizock, B. A. (2001). "Alterations in fuel metabolism in critical illness: hyperglycaemia." Best Pract Res Clin Endocrinol Metab 15(4): 533-51.

Patino, J. F., S. E. de Pimiento, et al. (1999). "Hypocaloric support in the critically ill." World J Surg 23(6): 553-9.

Prigeon, R. L., M. E. Roder, et al. (1996). "The effect of insulin dose on the measurement of insulin sensitivity by the minimal model technique. Evidence for saturable insulin transport in humans." J Clin Invest 97(2): 501-507.

Radziuk, J., T. J. McDonald, et al. (1978). "Initial Splanchnic Extraction of Ingested Glucose in Normal Man." MetabolismClinical and Experimental 27(6): 657669.

Rubinson, L., G. B. Diette, et al. (2004). "Low caloric intake is associated with nosocomial bloodstream infections in patients in the medical intensive care unit." Crit Care Med 32(2): 350-7.

Van den Berghe, G., P. Wouters, et al. (2001). "Intensive insulin therapy in the critically ill patients." N Engl J Med 345(19): 13591367.

Weissman, C. (1999). "Nutrition in the intensive care unit." Crit Care 3(5): R67-75.

Weitgasser, R., B. Gappmayer, et al. (1999). "Newer portable glucose meters Analytical improvement compared with previous generation devices?" Clinical Chemistry 45(10): 1821-1825. 